\title{
Painterly Assemblies: Making Through Scavenging
}

\author{
FAYSAL TABBARAH \\ American University of Sharjah
}

This paper presents an ongoing body of work that aims to disrupt prevailing Modernist tendencies within computational design practices and digital design methodologies as well as present an alternative for archaic and highly standardized modes of sustainable design production through describing the development of a painterly attitude towards digital and material computation and its resultant workflow. This ongoing body of work looks at the radical shift from the linear in late Renaissance to the painterly in the Baroque and its potential within the context of contemporary computational design methodologies and digital fabrication. The paper presents a workflow that includes scavenging for natural material, 3D scanning, along with digital and material assembly in the form of reciprocal frame systems.
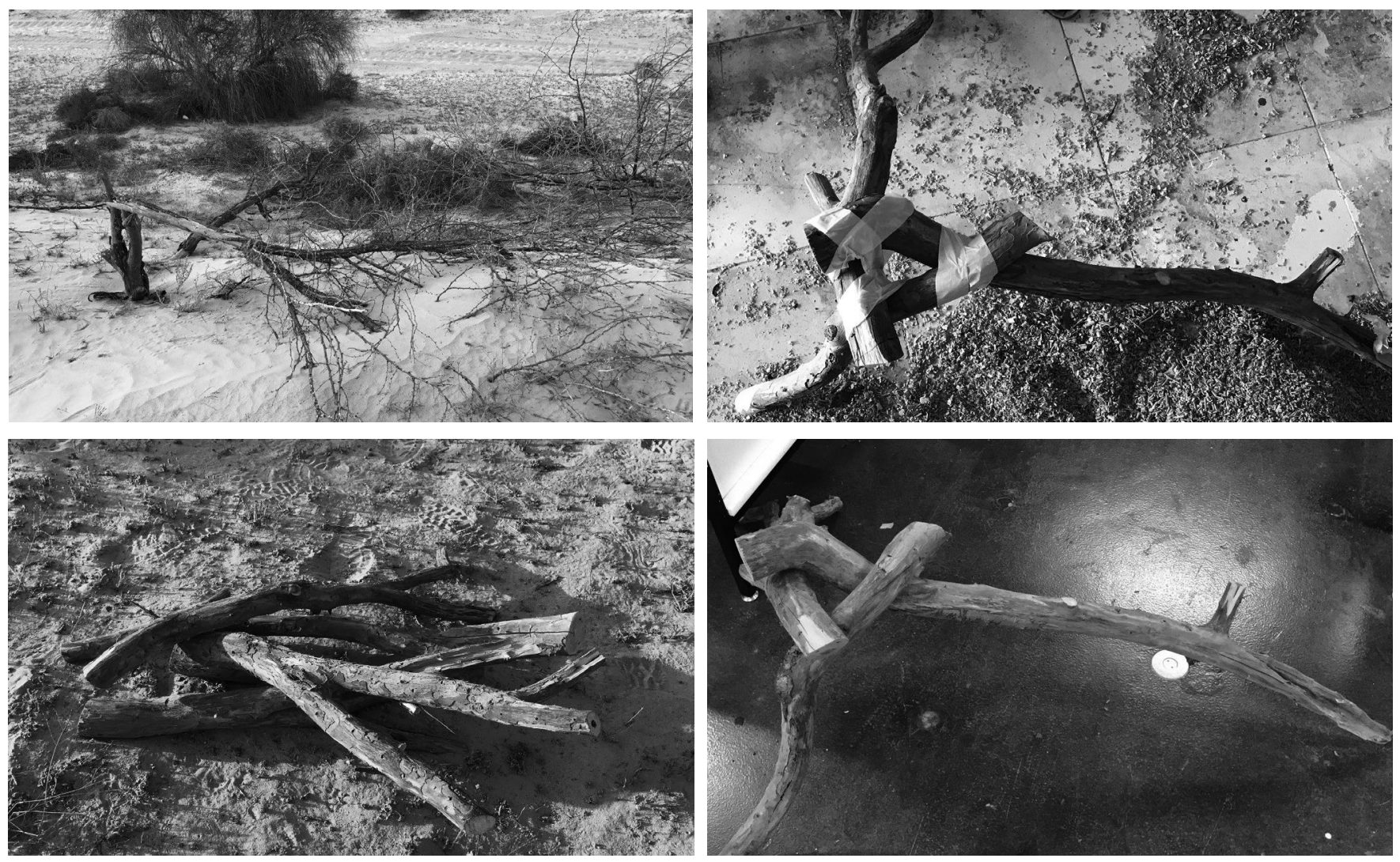

\author{
IBRAHIM IBRAHIM
}

American University of Sharjah

The paper is organized in three parts. First, it describes the relationship between the historical radical shift from the linear to the painterly and contemporary practices in computational design and digital fabrication. Second, it describes a digital-analog workflow that describes the relationships between scavenging for natural material, 3D scanning, a computational system and resultant material constructs that exhibit painterliness through its open system logic and blurring of traditional linear part-to-whole relationships. Third, it maps out a potential area of development in the near future for this evolving research and theoretical framework.

Figure 1: Shows the landscape that provides the raw material as well as possible timber configurations. 

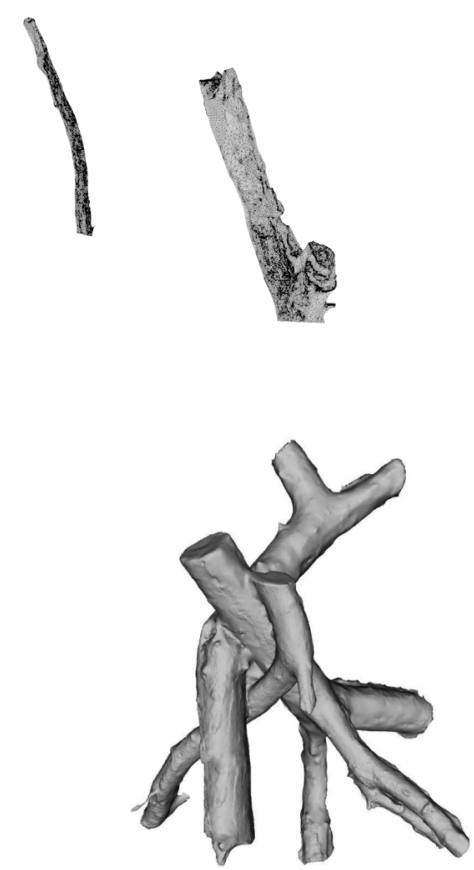

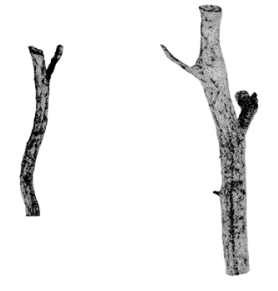

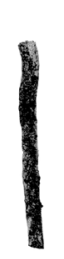
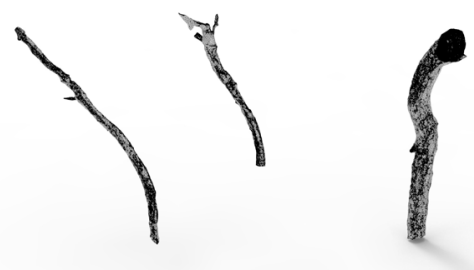
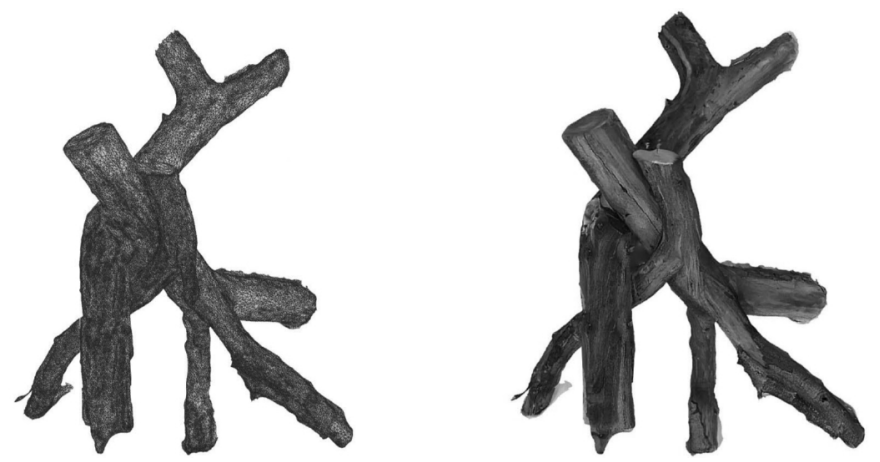

Figure 2: Digital model of self-supporting timber members.

\section{INTRODUCTION}

In his seminal work, Principles of Art History: The Problem of the Development of Style in Later Art, the Swiss art historian Heinrich Wofflin describes a radical shift in the development of European representational art styles between the LateRenaissance and the Baroque in sixteenth and seventeenth centuries. Specifically, Wofflin describes a conceptual, material and binary shift from the linear, to the painterly in the latter period. Wofflin's definition of the painterly, which he argues emerges in the early Baroque, alludes to ideas of limitlessness and merging, which he saw as standing in direct opposition to the tangibility and solidity of the Late Renaissance linear style. Wofflin describes the painterly as limitless, receding, open, lacking in hierarchy (i.e. Ambiguous part-to-whole relationships), and finally of relative clarity, in comparison to the absolute clarity found in Late-Renaissance ${ }^{1}$.

When looked at through a contemporary lens, it becomes apparent that the shift that occurred between the Late Renaissance and the Baroque, from the linear to the painterly, can potentially mirror the shifts between still-prevailing Modernist attitudes to architecture production and alternative modes of architecture production that are emerging today. However, this mirroring has not fully matured, and it is easy to identify that much of contemporary architecture production, specifically that which focuses on contemporary digital fabrication, operates in ways that are very Modernist, and by extension, share affinities with the Late Renaissance. In essence, contemporary practices focusing on digital fabrication can be identified as linear.

For example, the propagation of gradient surface patterns, made enormously easy by the democratization of off-theshelf computational design tools, belies a Modernist attitude towards flatness and frontality, unambiguous part-to-whole relationships, and absolute clarity. However, there is room to deploy Painterly attitudes within the context of computational design methodologies and digital fabrication.

It is within this space that this ongoing body of work operates. Specifically, there is an attempt to explore the potential of painterly attitudes within the context of contemporary computational design methodologies and digital fabrication techniques, to introduce material scavenging to create an open and limitless material system that blurs part-to-whole relationships in favor of ambiguous material hierarchies through the use of locally found fallen timber members and other synthetic materials.

\section{SCAVENGING}

This body of work situates itself within the context of masscustomization through the scavenging of fallen non-standard timber members to produce an architectural material system that exhibits structural as well as other performative 

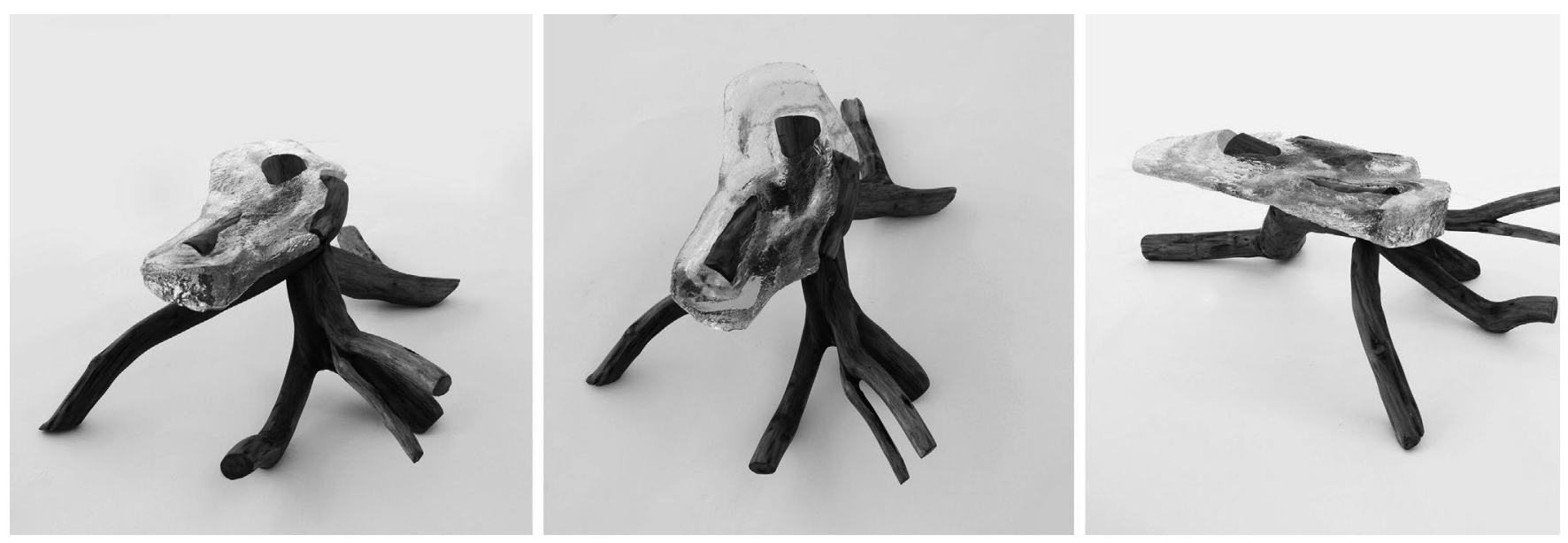

Figure 3: An iteration of the physical prototypes showing the resin-cast seat. This iteration aimed to make itself more invisible and structural. It locks itself through topologically designed holes in the volume that allow the timber to penetrate it.

characterists. Current work being produced within this vein include the ongoing research conducted at Architectural Association Design + Make programme, most recently in the form of Woodchip Barn, a shed structure in the form of a truss built by robotically cut and formed non-standard timber members. From a material resources point of view, the project adopts a scavenging attitude in the form of finding already fallen timber members within the deserts of the United Arab Emirates (figure 1). These are typically used for composting, and are therefore fully renewable resouserces and environmentally friendly. From a material assembly point of view, the project aims to avoid invasive approaches to the fallen timber member in the pursuit of larger structures. In essence, the project aims to use the material intelligence itself to create a structural condition without the use of any hardware, or at the very least, reduce the amount of hardware, and manufacturing in general, necessary. Thus, the project's structural principles are built on a self-supporting reciprocal frame typology that requires no mechanical or subtractive connections.

The scavenging process looks for four key criteria found in fallen timber members to better streamline the scavengingto-assembly workflow. The first criteria concerns the overall dimensions of the timber members. It was found that a significant nymber of the fallen timber members are around 70-80 linear centimeters, and $0.2 \mathrm{~m}^{3}$ in volume. The second criteria includes looking for particular cross sectional properties (approx. 7-15 centimeters in diameter). Thinner elements were initially explored in a bundled form, however, they were quickly discarded as that seemed redundant given the availability of bigger cross-sections. Third, the scavenging also looked for members that exhibited cross sectional strength due to natural occurring curvatures. The fourth and last criteria, which is the discernment of certain frictional properties of timber members due to natural weathering, dampness surface irregularities and textures at different scales have to be intuited from the various tests conducted throughout. These four properties allow for the development of a non-invasive reciprocal frame system (figure 1 , figure 2 , and figure 3 ). The scavenging process, while time consuming at first, serves two purposes. The first is that it provides relatively cheap raw material that also has the potential for an operation at an industrial scale if the list of stake holders expands. Moreoever, accepting that the raw material the material system operates with is non-standardized opens the doors for open systems that blur the painterly hierarchy between part and whole. The second reason for an allegiance to scavenging lies in its obvious economical and sustainable advantages, which follows a parallel agenda of disrupting traditional sustainable building design practices which have become extremely commercialized and standardized. In the long term, the research aims to build full scale architectural conditions and with that, it becomes important to assert that an alternative attitude towards green building practices that integrate natural, non-standardized elements with synthetic off-the-shelf or custom-made components offers potential within the context of the Anthropocene, climate change and emerging modes of movement of goods in ways that forces the discipline to disrupt some of its archaic practices towards novel and more valuable ways of making and conceiving of space.

\section{SCANNING FOR DIGITAL ASSEMBLY}

After the scavenging process, each found timber member is scanned using off-the-shelf 3D scanning software that compiles 3D meshes from a series of photographs. 3D Scanning allows for three key digital components of the process. First, scanning allows for quick sorting of elements given the three of the four criteria described above of dimensions, cross sectional radius, and overall curvature (figure 2 ). Second, a custom-designed digital workflow allows for matching same sized surfaces along the timber members based on a naturally occurring male-female joint (figure 3 ). This is then translated into the physical world with relative ease after developinga 


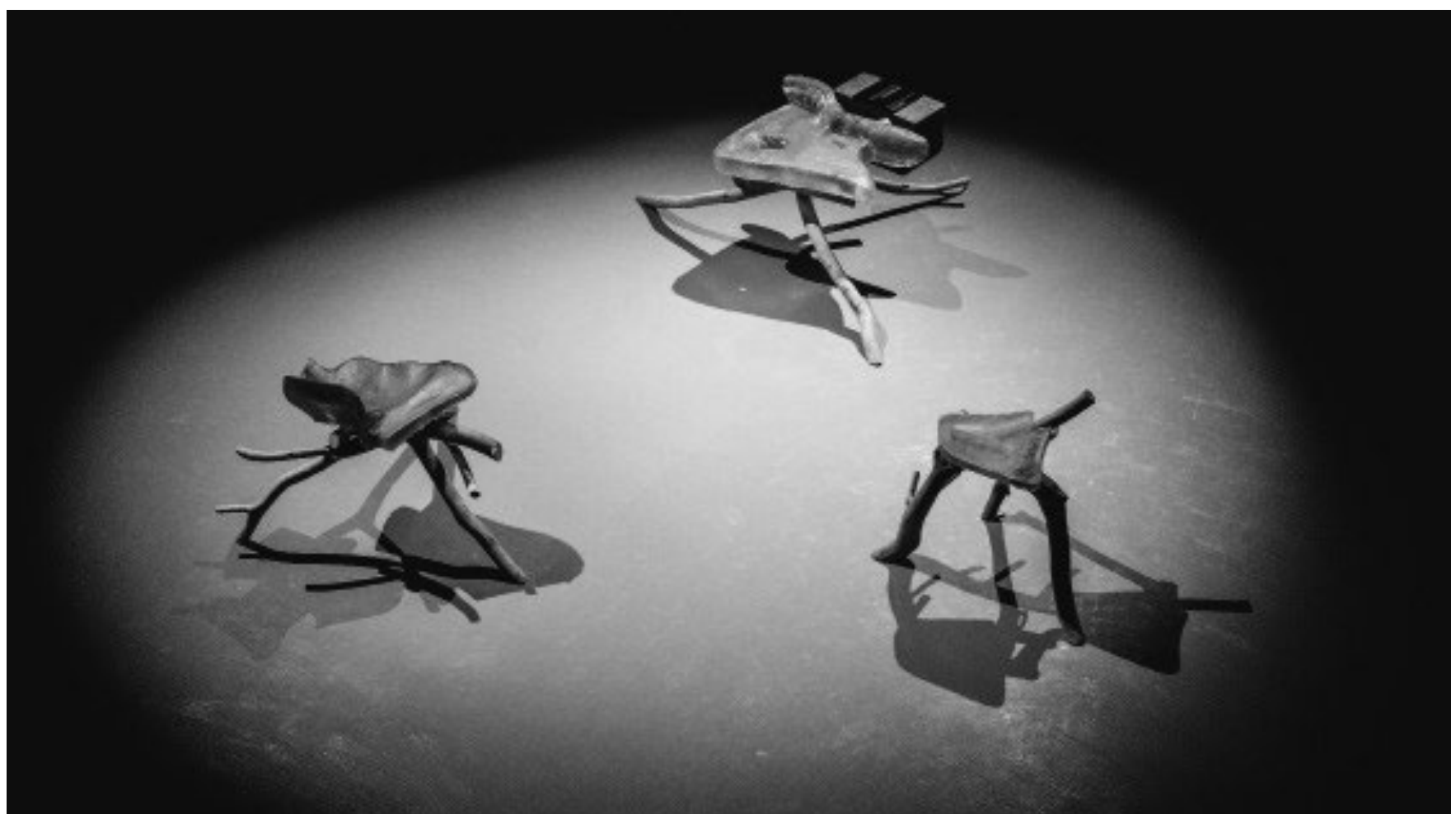

Figure 4: 3 chairs at full scale. Photo Credit: Amman Design Week

tagging system. The third and final reason for scanning is that it allows for the digital assembly of a reciprocal frame based on two performative conditions: 1 . Identifying matching male-female joints; 2 . Creating a stable reciprocal frame with at least three members that touch the ground on three points, thus existing within the invisible volume of a stable tetrahedron. These conditions are digitally "found" rather than "designed."

\section{PAINTERLY CHAIRS: INTEGRATED MATERIAL SYSTEMS}

Integrating the evolutionary model into the scavenging-3D scanning workflow required a test scenario. A chair was chosen for its relative ease of production from a scale point of view, but also because of the ability to scale the system up. The first step in designing a chair was to test the system under the basic load of a human being. figure 3 shows how a quick physical assembly of the scanned model from figure 2 was able to withstand a point load from a human standing on it without any extra mechanical supports. This moment within the research opened the door for scaling two-fold into the scale of a seat.

The advantage that creating a chair also brings is the potential ability to start integrating other material systems in order to take it one step forward into an architectural realm. Early investigations of integrating material systems included utilizing thinner twigs. While these exhibited extreme painterliness in that they blurred linear part-to-whole hierarchies and where complete open systems in that they can be amplified with other members, they failed to actually perform as a chair due to a lack of surface area provided for sitting. This prompted a research into the potential of introducing a material that provides enough surface area to create a seat, but also enforce the reciprocal system. The decision was made to create a volumetric transparent resin-cast condition that

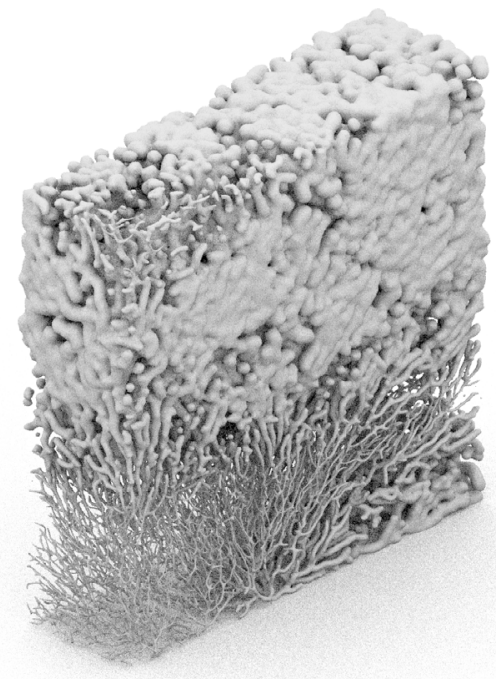

Figure 5: Developments of a wall system. 
hugs the timber members to lock them further from thrust. The first prototype did not perform strongly in that it kept slipping from its location, due to its form and slippery surface friction. This was resolved through redesigning the volume to have topological holes that allow for the timber to penetrate it and further lock the system into place.

\section{CONCULUSION AND FURTHER WORK}

In conclusion, the ongoing research presented in the paper aims to develop a painterly attitude towards digital and material computation to disrupt prevailing Modernist tendencies within computational design practices and digital design methodologies. Furthermore, this work also situates itself within the context of the sustainable building practices and it aims to present an alternative for archaic, highly standardized, codified and commercialized modes of sustainable building production through the introduction of natural building elements as legitimate building blocks.

Currently, this body of work is being developed on two different. First, the digital platform and workflow is being developed as a stand-alone package that would allow for existing 3D meshes to be input, sorted, matched and assembled in any location. This work looks for Enzo Mari's Autoprogettazione for inspiration with the added access to digital tools through smart phones. The second front of development aims to expand the scope of the material system to construct fullscale architectural elements such as walls or columns (figure 5 ) in a way that applies the theoretical framework to produce a spatial construct that puts the framework to task.

Acknowledgements: Portions of this work were exhibited in Amman Design Week 2017 with the credits a collaboration: Architecture + Other Things + Ibrahim Ibrahim.

\section{ENDNOTES}

1. Wolfflin, Heinrich. 1950. Principles of Art History: The Problem of the Development of Style in Later Art. Dover Publications.2.

2. Self, Martin and Emmanuel Vercruysse. 2017. "Infinite Variations, Radical Strategies," in Fabricate: Rethinking Design and Construction, edited A. Menges, B. Sheil, R. Glynn and M. Skavara. London: UCL Press. 30-35.2. 